\title{
Infertility insurance: What coverage exists for physician trainees?
}

Wade Muncey, MD; Erin Jesse, MD; Aram Loeb, MD; Nannan Thirumavalavan, MD

${ }^{1}$ Case Western Reserve University School of Medicine, Cleveland, OH, United States; ${ }^{2}$ University Hospitals Cleveland Medical Center, Cleveland, OH, United States

Cite as: Muncey W, Jesse E, Loeb A, et al. Infertility insurance: What coverage exists for physician trainees? Can Urol Assoc J 2020 December 15; Epub ahead of print. http://dx.doi.org/10.5489/cuaj.6824

Published online December 15, 2020

$* * *$

\section{Abstract}

Introduction: We aimed to describe infertility insurance coverage provided to male and female fellows working at institutions that offer advanced infertility training.

Methods: Faculty and fellows working within U.S. and Canadian Andrology or Reproductive Endocrinology and Infertility (REI) programs were contacted and asked for a copy of their institutional health insurance summary of benefits. Documents were assessed for coverage of diagnosis and treatment, shared costs, and maximum lifetime coverage for infertility care.

Results: Insurance policies from 24 institutions were reviewed; 16 of 24 (66\%) institutions covered costs related to the diagnosis of infertility. Six institutions $(25 \%)$ offered coverage for diagnosis but not treatment. There were 15 (62.5\%) institutions that offered some amount of coverage for the treatment of infertility, and the average lifetime maximum was $\$ 16100$. Only six of $24(25 \%)$ plans explicitly described a covered male-specific treatment, which included sperm extraction $(12.5 \%)$, varicocele repair $(4.2 \%)$, and sperm cryopreservation $(8.3 \%)$.

Conclusions: For physician trainees, infertility insurance coverage is not universal, policies are not transparent, and treatment for male factor infertility is often omitted. With high costs of infertility treatment, variable insurance coverage, and debt and time constraints, residents and fellows are a particularly vulnerable population that may experience significant financial toxicity when faced with infertility. 


\section{Introduction}

Infertility is defined as the inability to achieve pregnancy following 1 year of regular, unprotected vaginal intercourse, and can affect up to $15 \%$ of couples. ${ }^{1,2}$ Notably, the physician population may be at an increased risk for infertility. A recent study of 327 female physicians demonstrated an infertility rate of $24.1 \%$, nearly double the rate that of the general female population. ${ }^{3}$ An older age at time of pregnancy has been observed among female physicians, and may certainly contribute to this discrepancy, however, there may be additional factors at play. ${ }^{4}$ For example, women on shift work and rotating night shifts schedules, which are frequently encountered by physicians, have been shown to have higher rates of infertility. ${ }^{5}$ Prolonged work hours have been associated with increased miscarriages among female residents. ${ }^{6}$ The prevalence of infertility among male physicians is at this time unknown, however, shift work has also been associated with infertility, hypogonadal symptoms, and poor semen parameters in men. ${ }^{7}$ Among the general population, a male factor is responsible exclusively in $20 \%$ of infertility and cooccurs in another $30-40 \%{ }^{2}$

Infertility care is often expensive. A recent survey of 332 couples undergoing 18 months of evaluation for the diagnosis and treatment of both male and female infertility found that for medications, the average out-of-pocket expense was $\$ 912$, and increased to $\$ 2,623$ for intrauterine insemination. An even more substantial increase in cost was seen with in vitro fertilization (IVF), which averaged $\$ 19,234$ of out-of-pocket expense. ${ }^{8}$ Such a financial strain has been observed among many patients undergoing expensive medical treatments in the United States, and the term financial toxicity (FT) has been coined to describe the financial burden resulting from these high medical bills. ${ }^{9}$ Individuals facing infertility may fall victim to FT.

Physician trainees (residents and fellows) may be especially vulnerable to the economic burden of FT resulting from infertility. Residency and fellowship training often take place during peak years for childbearing, with the average age for incoming surgical residents reported as 27 years old. ${ }^{10}$ Furthermore, residents often have a high debt to income ratio. ${ }^{11}$ As healthcare workers, trainees faced with infertility typically look to their insurance plans for financial assistance. We therefore aimed to perform a descriptive study assessing general trends of insurance coverage for infertility care available to fellows within institutions that offer advanced physician infertility training. We hypothesize that the amount of insurance coverage available to physician trainees for the diagnosis and treatment of infertility is not congruent with high costs associated with such services.

\section{Methods}

As a sample of US and Canadian institutions with post-graduate physician training, we chose those with Andrology and Reproductive Endocrinology and Infertility (REI) fellowships, operating under the assumption that these institutions may provide high volume infertility care. These institutions were identified using the Society of Reproductive Endocrinology and 
Infertility (SREI) and the Society for the Study of Male Reproduction (SSMR) websites. Institutions' program websites and graduate medical education office websites were explored to obtain insurance plan summary of benefits. In addition, trainees (fellows) and faculty fellowships were contacted and asked to provide a copy of their institutional health insurance summary of benefits. Any institution for which the insurance policy information could not be obtained was excluded from the study.

The summary of benefits documents were individually examined for their descriptions of coverage for infertility services by two authors (WM and EJ), with any disagreement resolved by the corresponding author (NT). Our document search included coverage for diagnosis, treatment, descriptions of shared costs and maximum lifetime coverage. For institutions that offered different tiers of insurance policies (e.g. Tier $1-3$ ), we used the highest tier offered for our data acquisition. In addition, we assessed for inclusion of male-specific policies related to infertility care. Any diagnostic modalities and descriptions of therapies such as varicocelectomy, testicular and epididymal sperm retrieval, and microsurgical testicular sperm extraction, electroejaculation, penile vibratory stimulation and sperm cryopreservation were included as male-specific. An IRB approval was not required for this study.

\section{Results}

We obtained documents from 15 Andrology fellowship programs and 18 REI fellowship programs. There were 9 institutions with both andrology and REI programs, and thus we reviewed 24 unique institutions in total. There were 16 of $24(66 \%)$ institutions that covered costs related to the diagnosis of infertility. Seven (29\%) institutions did not include text regarding diagnostic coverage, and 1 explicitly excluded coverage for diagnosis. Fifteen out of the $24(62.5 \%)$ institutions reported any amount of coverage for the treatment of infertility. Six institutions (25\%) offered coverage for diagnosis but not treatment. There were 11 out of 24 $(45.8 \%)$ programs that explicitly covered IVF, and only $3(12 \%)$ of these policies specified coverage for sperm procurement. Among these 11 programs, only 3 included sperm procurement There were 12 (50\%) institutions covering fertility-specific medications. Only 6 out of 24 (25\%) plans explicitly described a covered male-specific treatment, which was sperm harvesting in 3 (12.5\%) cases, varicocele repair by 1 institution (4.2\%), and sperm cryopreservation was covered by $2(8.3 \%)$ programs. Cryopreservation was explicitly excluded from coverage in $8(33.3 \%)$ policies; the rest did not specify.

Of the institutions that provided coverage for treatment, the amount offered ranged from $50 \%-100 \%$ coverage of costs, with many specifying a lifetime maximum. Four $(26.6 \%)$ out of the 15 institutions reported $\geq 90 \%$ coverage of the treatment costs, whereas $5(33.3 \%)$ offered $<90 \%$ coverage; the rest did not specify. Among these 15 institutions that provided coverage for treatment of infertility, 13 reported the lifetime maximum amount, which averaged $\$ 16,100$ (range of $\$ 7,500$ - \$25,000). Of the 6 institutions that covered costs toward the diagnosis of 
infertility only, 5 reported their percent coverage, which ranged from $80 \%$ to $100 \%$. The last institution only reported a lifetime maximum of $\$ 2,500$ toward the cost of infertility diagnosis. These findings are summarized in Table 1.

\section{Discussion}

We present the first nationwide attempt to evaluate availability of fertility coverage for fellows. Our study underlines that among institutions that provide advanced infertility fellowship training, coverage for trainees is variable and not universal. These findings are of particular importance, as several recent studies have demonstrated that female physicians have substantially higher infertility rates than the general population. ${ }^{3,12}$ No study to date has investigated infertility among male physicians, but with half of all cases owing to a male factor, both sexes should have access to fertility services when trying to conceive. ${ }^{2}$ Postgraduate trainees have been shown to experience significant personal levels of stress, fatigue and burnout during their training years. ${ }^{13}$ There can be a multitude of relationship stressors at play as well, such as long work hours, frequent call and less time spent with a partner. ${ }^{14}$ In this backdrop, issues arising with infertility during this time period can become a significant emotional burden. Highlighting this effect, one study found that patients diagnosed with infertility experienced an emotional response comparable to a diagnosis of cancer. ${ }^{15}$ Despite infertility being defined as a disease by the World Health Organization, our study demonstrated that coverage does not always reflect this designation, particularly in programs that provide no coverage at all. ${ }^{16}$

Female physicians have been estimated to suffer from infertility at an incidence of 1 out of 4, and sadly, postponement of pregnancy among academic females was found to leave many "involuntarily childless" in one survey. ${ }^{3,17}$ One study illustrated this consequence, finding that among 113 female thoracic surgeons, the average age at first childbirth was 34 years, in comparison to the national average being $25 .{ }^{18}$ Our study focused on infertility coverage for trainees because this time frequently spans peak reproductive years, and insurance coverage of fertility care during this time period is of paramount importance. We found that among plans that provided insurance coverage, the average lifetime maximum for infertility treatment was $\$ 16,100$, but with some providing as little as $\$ 7,500$. Also, several plans that offered $\geq 90 \%$ coverage of total costs, capped their lifetime maximum amount well below the expenses associated with IVF. In evaluating published costs of female infertility treatment, it becomes apparent that these lifetime maximums are often inadequate. IVF may cost up to $\$ 20,000$ for one cycle, and if several cycles are required, the total cost can reach up to $\$ 100,000 .{ }^{19}$ For trainees with insufficient insurance coverage and fixed salaries, these costs can be prohibitive.

Insurance policies largely were inconsistent in their descriptions of covered services with a lack of generally agreed vocabulary. Diagnostic coverage was always reported without respect to gender and described vaguely as "procedures to determine the cause of infertility", "care, supplies and services leading to the diagnosis of Infertility", "infertility testing", "services to 
diagnose... the cause of Infertility." Descriptions of coverage for treatments offered were similarly vague without specific interventions described, except for those in reference to IVF services. For example, commonly used IVF procedure descriptions included: "uterine embryo lavage", "embryo transfer", "artificial insemination", "gamete intrafallopian tube transfer", "zygote intrafallopian tube transfer", "low tubal ovum transfer", "intracytoplasmic sperm injection", "oocyte retrieval", "frozen embryo transfer", "controlled ovarian hyperstimulation", "pronuclear stage transfer, and "oocyte retrieval". A similar level of specificity was absent for male factor infertility.

Despite recommendations that both male and female infertility workup should be completed at the onset, our study found a clear deficit in the description and coverage for services related to male infertility. ${ }^{20}$ Among all surveyed institutions, only $6(25 \%)$ had policies explicitly covering treatment for male factor infertility. Eight out of the 11 policies covering IVF failed to include sperm retrieval in their description of services. The only non-IVF related procedures related to male-factor infertility described were "varicocele repair" by one institution and "reversal of vasectomy" by another. These findings mirror a recent study by Dupree and colleagues that found that in 15 states with laws mandating insurance coverage for female factor infertility, only $8(53 \%)$ had text describing coverage for men. ${ }^{21}$

Out of the combined 24 institutions, only $3(12.5 \%)$ explicitly provided coverage for sperm harvesting, and $2(8.3 \%)$ provided coverage for cryopreservation. These findings are alarming because they indicate that at many institutions, male infertility treatment and subsequent cryopreservation are out-of-pocket expenses. A 1997 study found that following microsurgical sperm extraction and subsequent intracytoplasmic sperm injection, the cost per newborn totaled $\$ 51,024 .{ }^{22}$ Costs for male infertility services were more recently evaluated in a 2016 retrospective study. The authors found that out of 111 participants, $64 \%$ spent over $\$ 15,000$ and $47 \%$ professed financial strain from infertility related costs. ${ }^{23}$ Our evaluation found that only $1(4.2 \%)$ infertility policy included varicocele repair in the description of covered services, despite there being several studies demonstrating cost saving benefits of varicocelectomy when compared to other assisted reproductive technologies (ART). ${ }^{24,25}$ Providing this coverage could potentially alleviate the burden of more invasive treatments on the female partner and possibly allow for a spontaneous pregnancy. ${ }^{26}$

Finally, though FT is commonly used to describe the fiscal and personal hardship of cancer-related treatments, we would propose the term could be applied to patients undergoing infertility treatment as well. In 2018, the median debt upon entering residency was $\$ 194,000$, and in 2019 the average salary was $\$ 61,200 .{ }^{27,28}$ With this level of debt to income ratio, it would appear obvious that the costs of ART are not manageable without insurance coverage. Our findings show that infertility coverage is not universal, often would not cover average costs for 1 cycle of IVF ${ }^{29}$ and treatment of male factor infertility is largely absent from the majority of insurance policies. Furthermore, when considering the average work week of surgical trainees is 
84 hours, ${ }^{30}$ finding protected time for infertility treatment is difficult to achieve. A recent national survey evaluating the experience of infertility among resident physicians echoed these findings. For those with infertility, forgoing treatment due to "not having time" and "not being able to afford treatment" made up $60 \%$ of the survey responses. ${ }^{31}$

Readers must be cautioned that while this study provides insight into fertility coverage among physician trainees, there are significant limitations. We obtained insurance coverage documents from less than $50 \%$ of female fertility fellowships and we must acknowledge that our overall results may suffer from sampling bias. For example, participants from programs with strong fertility coverage or who have experienced infertility themselves may be more apt to provide information. We must also acknowledge that despite the majority of policies omitting varicocele repair as an infertility treatment, it does not necessarily mean a lack of coverage. Varicocelectomy may be included as a covered service for other indications beyond the treatment of male factor infertility, or it may simply be covered under the category of "outpatient surgery", for example. In addition, our methodology did not account for "unofficial understandings" or "policies" in which fellows may have different cost-saving arrangements, which one program did relay to us. Given the vague language that was frequently used in these policies, we may have underestimated the actual coverage that would be provided on a case-bycase basis. Lastly, the total number of institutions evaluated in this study were small and may not be generalizable to all training programs. Future studies with surveys dispersed nationally among all physician trainees would likely provide a more comprehensive representation of fertility coverage for trainees.

\section{Conclusions}

This study examined the incidence and descriptions of insurance coverage for infertility provided to physician trainees nationwide at institutions where advanced infertility training is offered. We found that infertility insurance coverage is not universal, policies are not transparent, and coverage for treatment of male factor infertility is typically omitted or minimally mentioned. With high costs of infertility treatment, variable insurance coverage, debt and time constraints, trainees represent a particularly vulnerable population that may frequently experience financial toxicity when faced with fertility concerns. 


\section{References}

1. Definitions of infertility and recurrent pregnancy loss: a committee opinion. Fertil Steril 2020 .

2. Thonneau $\mathrm{P}$, Marchand S, Tallec A, et al: Incidence and main causes of infertility in a resident population (1 850 000) of three french regions (1988-1989). Hum Reprod 1991.

3. Stentz NC, Griffith KA, Perkins E, et al: Fertility and Childbearing among American Female Physicians. J Women's Heal 2016.

4. Lerner LB, Stolzmann KL, Gulla VD. Birth trends and pregnancy complications among women urologists. J Am Coll Surg 2009.

5. Fernandez RC, Marino JL, Varcoe TJ, et al. Fixed or Rotating Night Shift Work Undertaken by Women: Implications for Fertility and Miscarriage. Semin Reprod Med 2016.

6. Klebanoff M.A., Shiono P.H., and Rhoads G.G.: Outcomes of pregnancy in a national sample of resident physicians. $N$ Engl J Med 1990.

7. Deng N, Kohn TP, Lipshultz LI, Pastuszak AW. The Relationship Between Shift Work and Men's Health. Sex Med Rev 2018.

8. Wu AK, Odisho AY, Washington SL, et al: Out-of-pocket fertility patient expense: Data from a multicenter prospective infertility cohort. J Urol 2014.

9. Casilla-Lennon MM, Choi SK, Deal AM, et al: Financial Toxicity among Patients with Bladder Cancer: Reasons for Delay in Care and Effect on Quality of Life. J. Urol 2018.

10. Stain SC, Hiatt JR, Ata A, et al: Characteristics of highly ranked applicants to general surgery residency programs. JAMA Surg 2013.

11. Tevis SE, Rogers AP, Carchman EH, et al: Clinically Competent and Fiscally at Risk: Impact of Debt and Financial Parameters on the Surgical Resident. J. Am Coll Surg 2018.

12. Gyorffy Z, Dweik D and Girasek E: Reproductive health and burn-out among female physicians: Nationwide, representative study from Hungary. BMC Womens Health 2014.

13. Jennings ML and Slavin SJ: Resident wellness matters: Optimizing resident education and wellness through the learning environment. Acad Med 2015.

14. Finch SJ: Pregnancy during residency: A literature review. Acad Med 2003.

15. Domar AD, Zuttermeister PC and Friedman R: The psychological impact of infertility: A comparison with patients with other medical conditions. J Psychosom Obstet Gynaecol 1993.

16. Zegers-Hochschild F, Adamson GD, de Mouzon J, et al: International Committee for Monitoring Assisted Reproductive Technology (ICMART) and the World Health Organization (WHO) revised glossary of ART terminology. Fertil Steril 2009.

17. Kemkes-Grottenthaler A: Postponing or rejecting parenthood? Results of a survey among female academic professionals. J Biosoc Sci 2003.

18. Pham DT, Stephens EH, Antonoff MB, et al: Birth trends and factors affecting childbearing among thoracic surgeons. Ann Thorac Surg 2014.

19. Marshall AL, Arora VM and Salles A: Physician Fertility: A Call to Action. Acad Med 2020; 95: 679-681.

20. Diagnostic evaluation of the infertile male: A committee opinion. Fertil Steril 2012. 
21. Dupree JM, Dickey RM and Lipshultz LI: Inequity between male and female coverage in state infertility laws. Fertil Steril 2016.

22. Kolettis PN and Thomas AJ: Vasoepididymostomy for vasectomy reversal: A critical assessment in the era of intracytoplasmic sperm injection. J Urol 1997.

23. Elliott PA, Hoffman J, Abad-Santos M, et al: Out-of-Pocket Costs for Men Undergoing Infertility Care and Associated Financial Strain. Urol Pract 2016.

24. Penson DF, Paltiel AD, Krumholz HM, et al: The cost-effectiveness of treatment for varicocele related infertility. J Urol 2002.

25. P.N. S: Is assisted reproduction the optimal treatment for varicocele- associated male infertility? A cost-effectiveness analysis. Urology 1997.

26. Çayan S, Erdemir F, Özbey I, et al: Can varicocelectomy significantly change the way couples use assisted reproductive technologies? J Urol 2002.

27. Kothari P, Gilbert J, Sánchez N, et al: Financing a Career in Academic Medicine. In: Succeeding in Academic Medicine. Springer 2020; pp 149-164.

28. Medscape Residents Salary \& Debt Report 2019. Available at: https://www.medscape.com/slideshow/2019-residents-salary-debt-report-6011735\#2, accessed May 12, 2020.

29. Strasser MO and Dupree JM: Care Delivery for Male Infertility: The Present and Future. Urol Clin North Am 2020; 47: 193-204.

30. Mendelsohn D, Despot I, Gooderham PA, et al: Impact of work hours and sleep on wellbeing and burnout for physicians-in-training: the Resident Activity Tracker Evaluation Study. Med Educ 2019.

31. Aghajanova L, Hoffman J, Mok-Lin E, et al: Obstetrics and Gynecology Residency and Fertility Needs. Reprod Sci 2017.

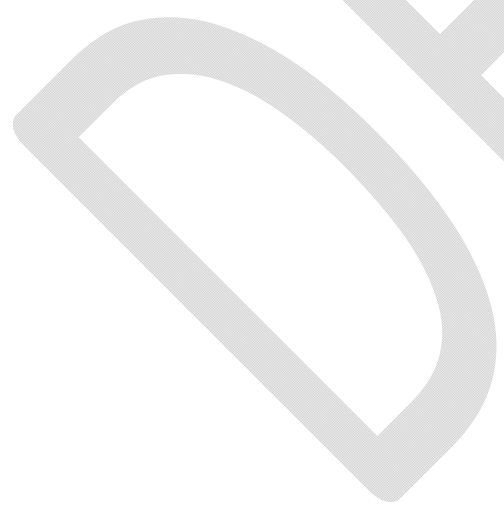




\section{Figures and Tables}

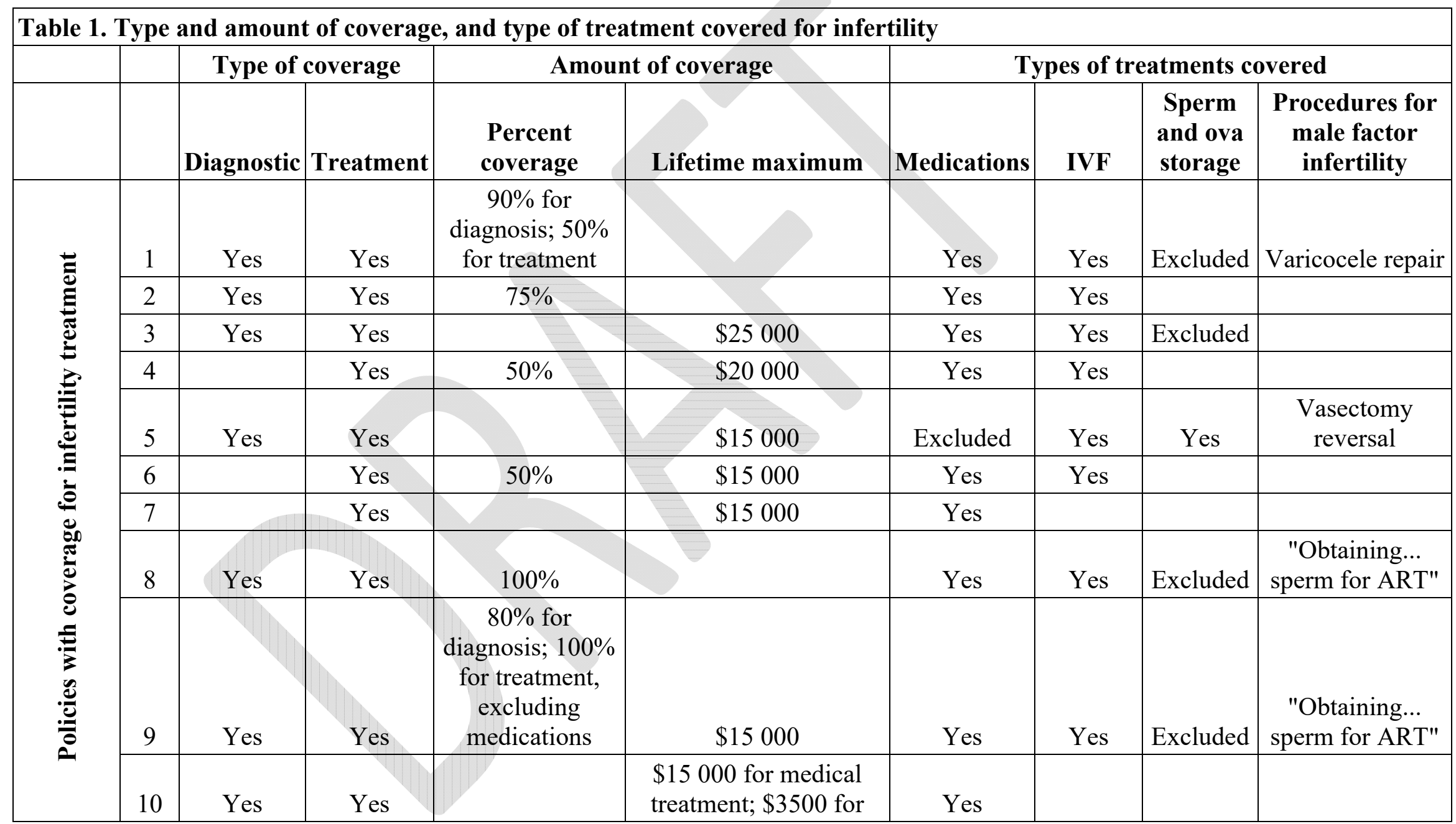




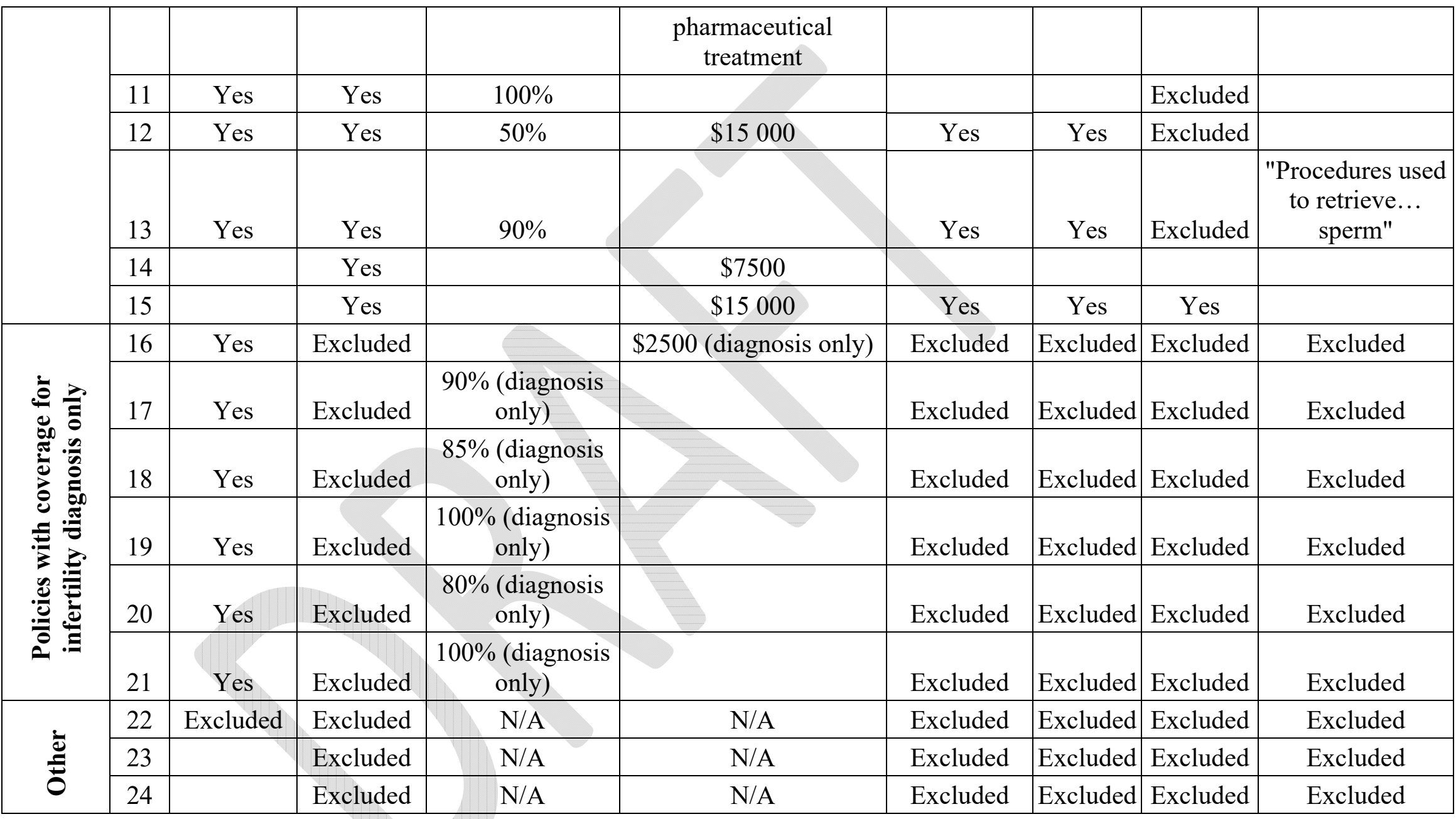

Blank cells denote a "not specified" response. 